\title{
Report of a Case: Pseudoaneurysm of the Cystic Artery With Hemobilia Treated by Arterial Embolization
}

\author{
Yoshihiro Komatsu ${ }^{\mathrm{a}}$, Hajime Orita ${ }^{\mathrm{a}}$, Mutsumi Sakurada ${ }^{\mathrm{a}}$, Hiroshi Maekawa ${ }^{\mathrm{a}}$, \\ Toshitaka Hoppo ${ }^{\mathrm{b}}$, Koichi Sato ${ }^{\mathrm{a}, \mathrm{c}}$
}

\begin{abstract}
We report a case of hemobilia caused by pseudoaneurysm of the cystic artery in a 71-year-old woman who presented with fever and epigastric colicky pain with jaundice. Liver function tests showed signs of obstructive jaundice. On the second day, patient had a massive hematemesis and melena with hypovolemic shock. Hemobilia was diagnosed by endoscopically visualizing bleeding from the papilla of Vater. An emergent angiography demonstrated the presence of a pseudoaneurysm in the cystic artery. Selective embolization of the cystic artery was then performed to interrupt the blood flow into the pseudoaneurysm. Immediately after embolization, patient was hemodynamically stabilized. Although the patient did not undergo cholecystectomy after embolization due to severe co-morbidities, no signs of ischemic gallbladder have been observed. Hemobilia should be included in the differential diagnosis of upper gastrointestinal bleeding with unknown etiology. Embolization could be an option for pseudoaneurysms of the cystic artery especially in highrisk patients.
\end{abstract}

Keywords: Hemobilia; Pseudoaneurysm; Non-traumatic; Transcatheter arterial embolization

\section{Introduction}

Hemobilia, which is defined as bleeding into the biliary

Manuscript accepted for publication June 23, 2011

${ }^{\text {a }}$ Department of Surgery, Juntendo Shizuoka Hospital, Juntendo University School of Medicine. 1129 Nagaoka, Izunokuni-shi, Shizuoka, 410-2295, Japan

${ }^{\mathrm{b}}$ Department of Cardiothoracic Surgery, University of Pittsburgh Medical Center. Suite 715, 5200 Centre Avenue, Pittsburgh, PA, 15232, USA

'Corresponding author: Koichi Sato. Email: kou-sato@chive.ocn.ne.jp

doi:10.4021/jmc249w tree, is a rare entity and was first described by Sandblom in 1948 [1]. Hemobilia occurs when there is a communication between a vessel and the intrahepatic or extrahepatic biliary system and is typically represented as Quincke's triad: colicky abdominal pain, jaundice, and gastrointestinal hemorrhage [2]. The etiology of hemobilia includes iatrogenic trauma, accidental trauma, gallstones, inflammation, vascular malformations and tumors. Accidental trauma used to be the major cause of hemobilia. Currently, two-thirds of hemobilia are caused by iatrogenic trauma, which has been rapidly increasing with the frequent applications of percutaneous hepatic and biliary procedures such as liver biopsy and percutaneous biliary drainage [2]. In contrast, non-traumatic hemobilia due to inflammation, gallstone, and vascular malformations are extremely rare and also difficult to be diagnosed compared to traumatic hemobilia. Pseudoaneurysm of the cystic artery is one of non-traumatic hemobilia and as few as 22 cases have been reported [3-24]. In most of these cases, the patients were treated by either primary cholecystectomy or transcatheter arterial embolization (TAE) followed by cholecystectomy.

We report a case of hemobilia caused by psuedoaneurysm of the cystic artery which was successfully treated by performing TAE with metallic coils, without the following cholecystectomy.

\section{Case Report}

A 71-year-old Japanese woman was sent to the Emergency Department (ED) at our institution, with a subacute onset of epigastric abdominal pain originating from the right upper quadrant. The patient denied a history of hematemesis and melena. Past medical history included hypertension and Parkinson's disease. She has been suffered by Parkinson's disease for more than 20 years. The control of this disease has been getting worse and she could hardly walk by herself. She has spent most of her time on wheelchair or lying in bed.

On admission, conjunctiva was slightly pale and icteric. Blood pressure was $90 / 56 \mathrm{mmHg}$, body temperature was $38.7^{\circ} \mathrm{C}$, respiratory rate was $20 / \mathrm{min}$, and heart rate was $90 /$ $\mathrm{min}$. The epigastric region appeared full and was tender with 


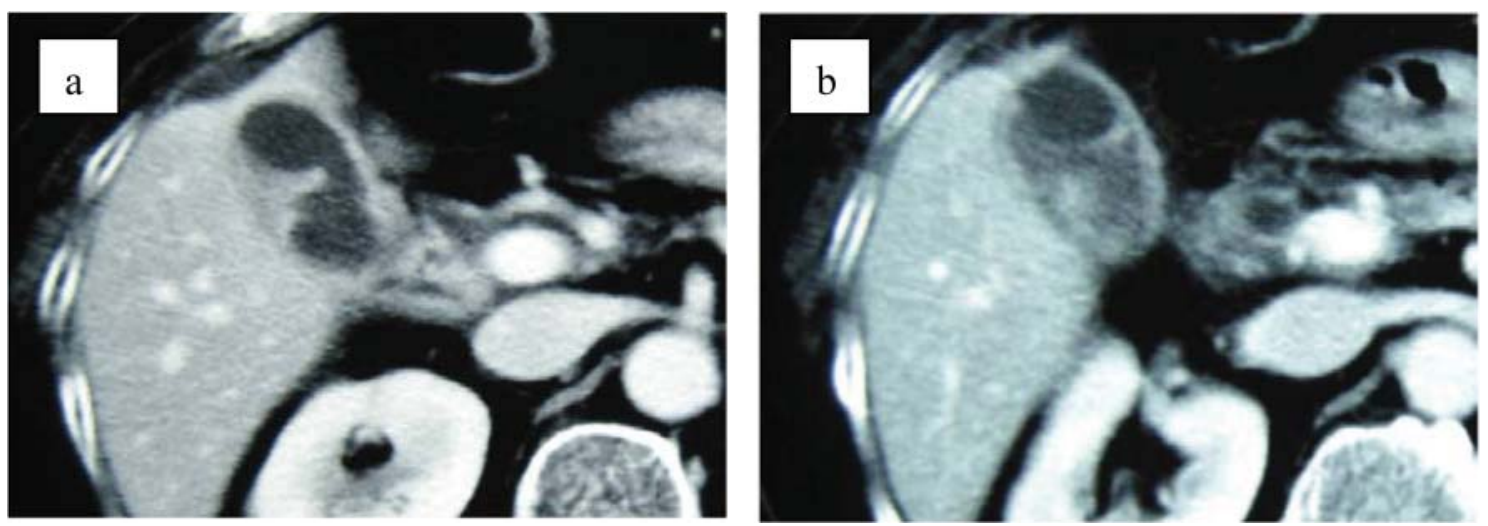

Figure 1. (a) Contrast-enhanced computed tomography shows thickening of gallbladder wall. (b) Contrast-enhanced CT shows enhanced lesion in the gallbladder. No structure of pseudoaneurysm is suspected.

palpation. There were no obvious peritoneal findings such as rebound tenderness. Rectal examination was negative for melena. She spoke in very soft voice and joints were stiff because of Parkinson's disease. Laboratory data on admission showed that white blood cell count $(7600 / \mu \mathrm{L})$, hemoglobin $(12.5 \mathrm{~g} / \mathrm{dl})$ and platelet count $\left(16.6 \times 10^{4} \mu \mathrm{L}\right)$ were normal. Liver function tests showed that total bilirubin $(6.1 \mathrm{mg} / \mathrm{dl})$, alkaline phosphates $(913 \mathrm{IU} / \mathrm{L})$, aspartate aminotransferase (AST) (329 IU/L), alanine aminotransferase (ALT) (343 IU/L), $\gamma$-glutamyl transpeptidase (410 IU/L), lactate dehydrogenase (LDH) (252 IU/L) and C-reactive protein (CRP) $(5.1 \mathrm{mg} / \mathrm{dl})$ were elevated. Coagulation tests were normal.

Although abdominal ultrasonography (US) was immediately performed at ED, it was not diagnostic because of difficulty to hold the proper posture due to Parkinson's disease and excessive bowel gas. Contrast-enhanced computed tomography (CT) showed marked thickening of the gallbladder wall, which contained high density material. However, these findings did not suggest the presence of vascular abnormalities such as aneurysm (Fig. 1). Magnetic resonance cholangiopancreatography (MRCP) showed two of approximately $15 \mathrm{~mm}$ sized circular signal defects in gallbladder in conjunction with dilatation of biliary tree and two or three 5 to $8 \mathrm{~mm}$ sized signal defects in the common bile duct close to the papilla of Vater (Fig. 2). Radiologist suggested that there are possibly stones in gallbladder and common bile duct at this point. Based on the radiographic findings, differential diagnosis included acute cholicystitis, cholelithiasis, and choledocholithiasis at this point. Fluid resuscitation and antibiotics were started to treat this condition.

On the second day of admission, even though the clinical symptoms and the signs of inflammation appeared to be
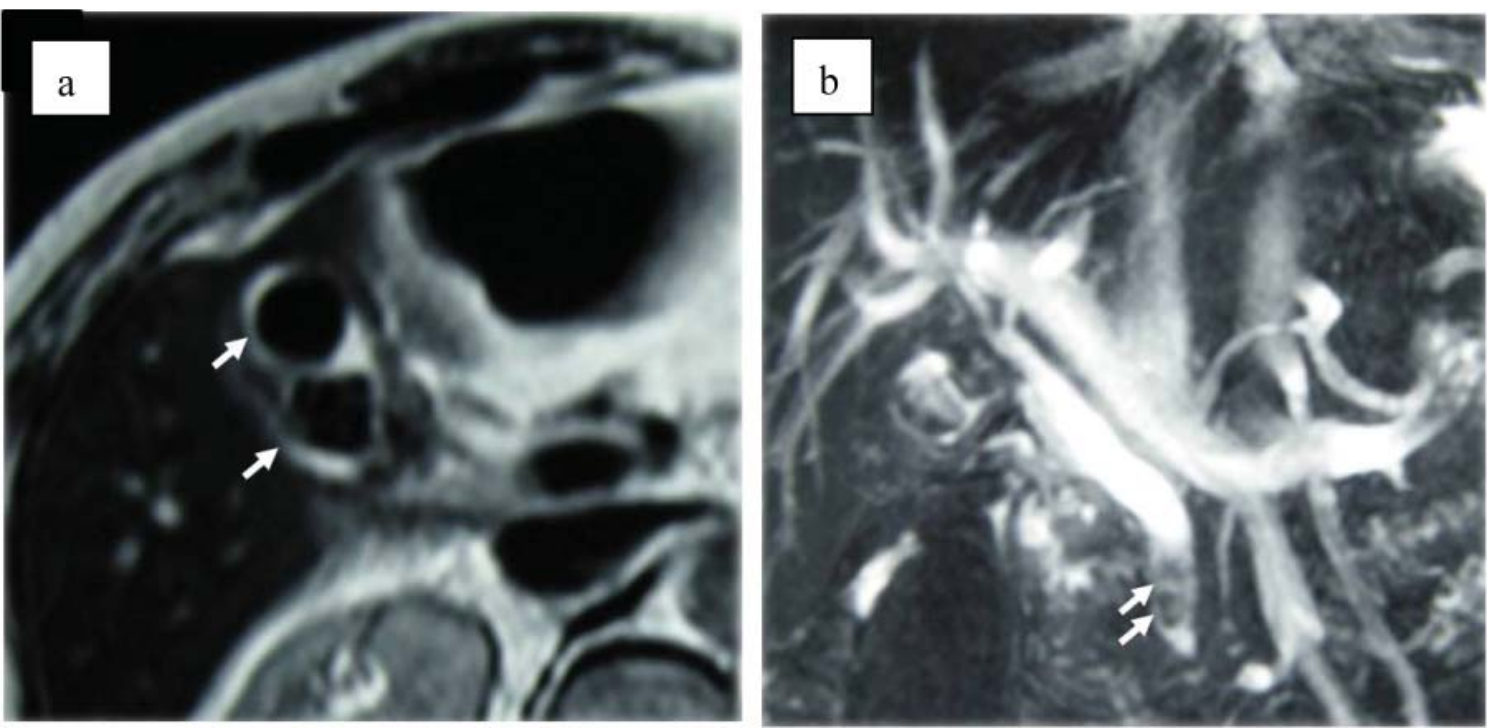

Figure 2. (a) MRI suspects two stones in the gall bladder (arrows). (b) MRCP suspects clots or stones in common bile duct (arrows) 


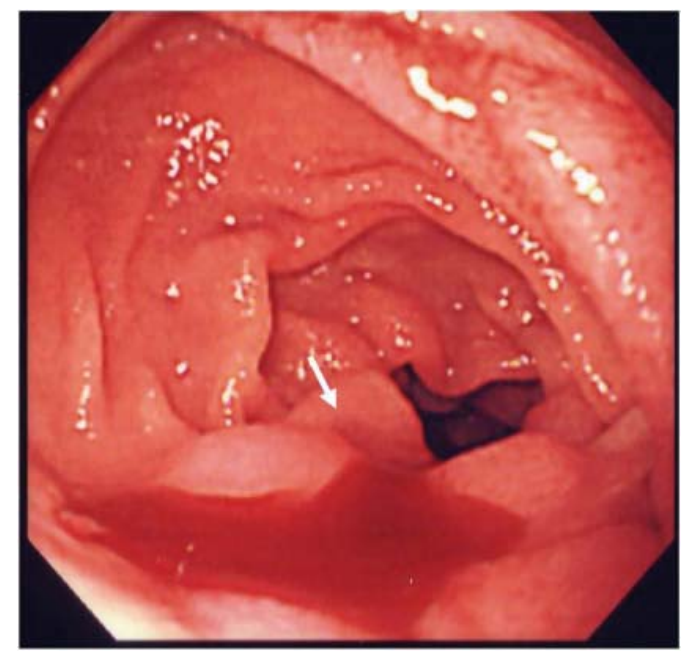

Figure 3. Endoscopy demonstrates blood oozing from the papilla of Vater (arrow).

improving, the patient vomited a large amount of coagulated blood and digital examination revealed a large amount of tarry stool in the rectum. This was the first as well as only episode of hematomesis and melena during the entire clinical course. However, the amount of blood vomited was estimated at approximately $500 \mathrm{~mL}$ and the patient showed hypovolemic shock with low systemic blood pressure of un- der $80 \mathrm{mmHg}$ and tachycardia of $100 / \mathrm{min}$. The patient was given 2 units of red blood cells transfusion and rapid ringer infusion for resuscitation. In the meantime, emergent upper endoscopy was performed and demonstrated a large amount of coagula in the stomach. No obvious site of bleeding was found in the stomach after removal of the coagula. Upper endoscopy was then advanced to the 2 nd portion of duodenum demonstrating that a small amount of blood was suddenly spouted out from the papilla of Vater (Fig. 3). This finding was observed only once during the procedure, although we kept on observing there for more than 30 minutes. At this point, decision was made to perform dynamic CT to evaluate the cause of hemobilia. The arterial and portal venous phases of dynamic CT showed the presence of approximately 12 $\mathrm{mm}$ nodular lesion at possibly inside the neck of gall bladder with an enhanced pattern parallel to the arterial phase in conjunction with the thickening of gallbladder wall (Fig. 4). The nodular lesion, which was not detected on admission, showed no obvious extravasation of contrast agents into surrounding structures. These results suggested the presence of newly developed vascular pseudoaneurysm in conjunction with possible cholecystitis. Although the dynamic CT did not demonstrate any signs of active bleeding from the nodular lesion, patient was hemodynamically unstable and digital subtraction angiography (DSA) was performed for the diagnostic and therapeutic purpose. At the time of DSA,
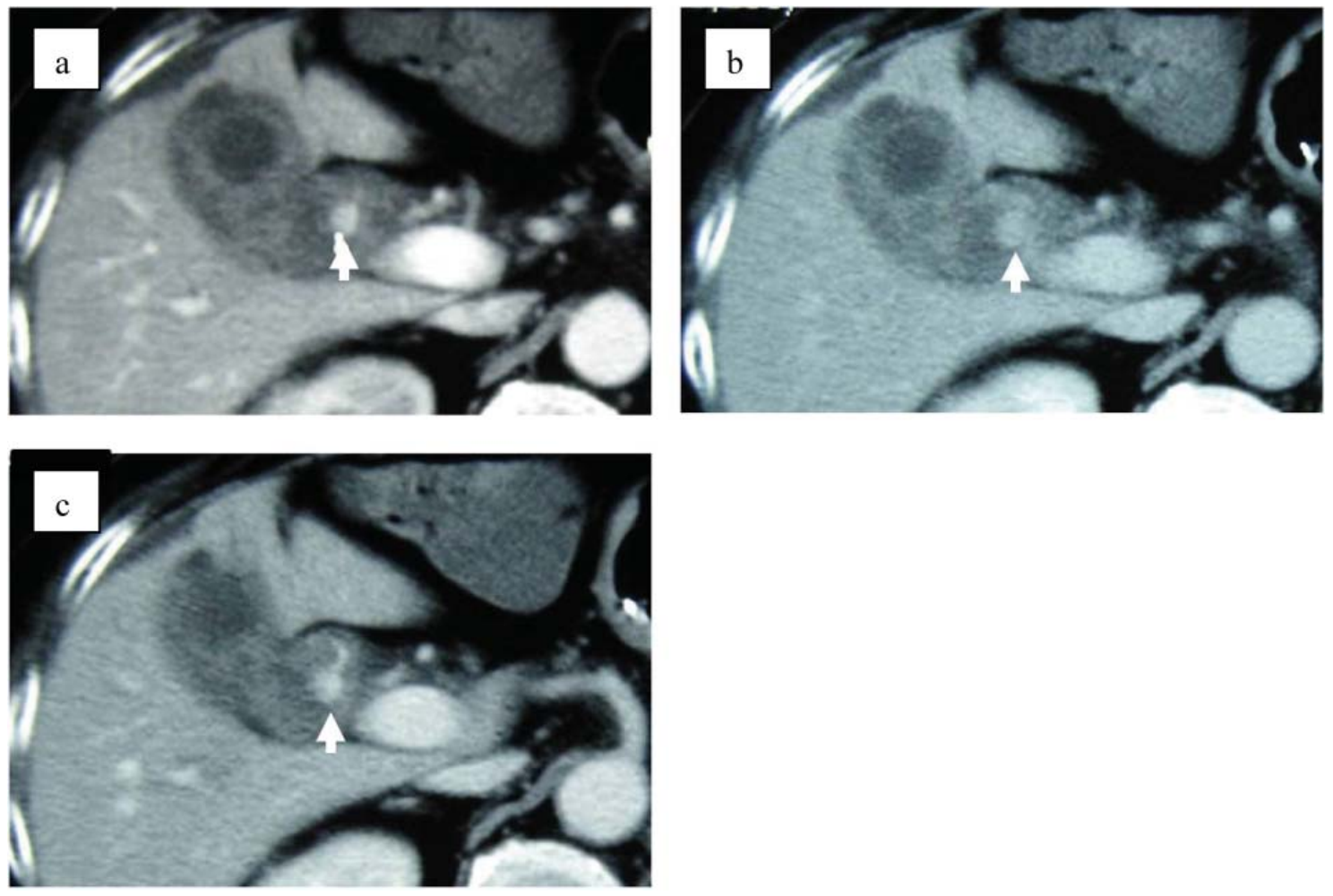

Figure 4. Dynamic CT shows $12 \mathrm{~mm}$ nodular lesion at the neck of gall bladder with an enhancement pattern parallel to the artery (arrows). (a) Arterial phase. (b) Balance phase. (c) Portal venous phase. 

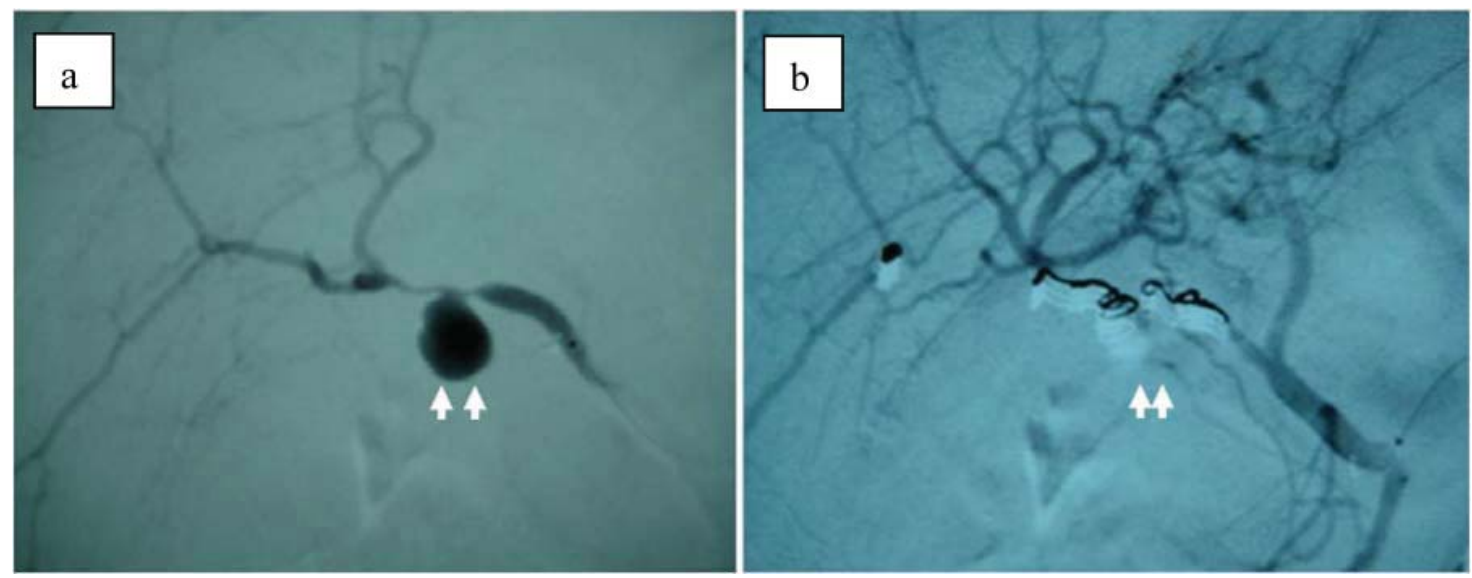

Figure 5. (a) Selective right hepatic angiography shows a pseudoaneurysm (arrow) of the cyctic artery. (b) After embolization with microcoils, arterial inflow to the cystic artery is completely blocked.

therapeutic materials such as micro coils and gelform were ready to use for the treatment of unexpected bleeding. DSA through the hepatic artery demonstrated the presence of a pseudoaneurysm originated from the cystic artery without any extravasation of contrast agents (Fig. 5a). Although there was no sign of active bleeding into biliary tree, it was thought that the intermittent bleeding due to the rupture of pseudoaneurysm could cause hemobilia. The right hepatic artery was selectively catheterized, and one microcoil was then placed distal and the other one was placed proximal to the aneurysm to block the arterial inflow to the pseudoaneurysm. Prior to embolization, we confirmed that the sufficient blood supply to the right liver lobe was preserved through the left hepatic artery (Fig. 5b).

The patient was hemodynamically stabilized immediately after embolization and the clinical symptoms improved significantly. Based on further assessment by anesthesiologists, decision was made not to proceed to any further surgical management such as cholecystectomy because of her severe co-morbidities, and also the patient refused to undergo surgery. Endoscopic retrograde cholangiopancreatography (ERCP) demonstrated the presence of stones in the gallbladder, and following the balloon dilation of the sphincter of Odd, small stones came out from the papilla of Vater. At a follow-up of 12 months, the patient was asymptomatic and satisfied with the outcome of treatment.

\section{Discussion}

Non-traumatic cause of hemobilia includes inflammatory conditions such as cholecystitis and acute pancreatitis, and malignancies. Twenty-two cases of hemobilia caused by pseudoaneurysm of the cystic artery have been published in English literature. In most of these cases, abdominal US were performed as a primary imaging modality to screen the cause of abdominal pain. In 6 cases, pseudoaneurysm of the cystic artery was suspected by color doppler US and followed by enhanced and/or dynamic CT, dynamic MRI, and/or angiography to confirm the diagnosis. Enhanced CT would be the second imaging modality to detect the pseudoaneurysm. Following the initial evaluation with abdominal US and CT, angiography was then performed in most of cases to visualize the vascular abnormalities such as psuedoaneurysm and 8 cases subsequently underwent TAE. Of 18 cases, in which gastrointestinal bleeding such as hematemesis or melena occurred, the blood from papilla of Vater was successfully observed in 6 cases under direct endoscopic visualization and hemobilia was confidently diagnosed. As the present case showed, the direct observation of hemobilia from papilla of Vater has been difficult to encounter, although it is crucial for the accurate diagnosis. Based on the diagnosis of hemobilia, 15 cases were treated with surgery, 3 cases with TAE followed by surgery, and 4 cases with only TAE. The 4 cases, which were treated with only TAE, were not complicated by gallbladder ischemia.

A pseudoaneurysm is a hematoma that is formed as the result of blood leaked from injured arterial wall. The hematoma develops outside the arterial wall so it is wrapped by the surrounding tissues. The exact mechanism to develop a pseudoaneurysm and the association between pseudoaneurysm and inflammatory process such as cholecystitis remains unclear. In the review of 16 cases of cystic artery pseudoaneurysm caused by cholecystitis, Akatsu et al [8] assumed that the acute inflammation of the gallbladder wall may cause both ulceration of serosa and partial erosion of the elastic and muscular components of the arterial wall, thus leading to the formation and rupture of the pseudoaneurysm. The presence of gall stone may accelerate the formation of the pseudoaneurysm. Furthermore, arterial sclerosis due to hypertension is considered as a risk factor of aneurysm formation [25] and weakness of arterial wall due to arterial sclerosis may also accelerate the formation of the pseudoaneurysm.

In the present case, the pseudoaneurysm of the cystic 
artery was not detected by US, enhanced CT, or MRCP. One of the signal defects in gall bladder in MRCP could be the pseudoaneurysm, however angiography showed that the pseudoaneurysm was present at the different location, the neck of gall bladder. Although initial enhanced CT did not show any sign of vascular abnormalities, dynamic CT clearly demonstrated the presence of psuedoaneurysm two days after admission. The inflammatory process on gallbladder may be associated with the formation of pseudoaneurysm, which may be developed or enlarged after admission. On the other hand, initial enhanced CT was sliced every $10 \mathrm{~mm}$ meanwhile dynamic CT was sliced every $7 \mathrm{~mm}$. The size of aneurysm measured by DSA was approximately $15 \mathrm{~mm}$ of diameter so that initial enhanced CT may have missed the initiation of pseudoaneurysm formation.

Although the typical presentation of hemobilia is represented by Quincke's triad such as colicky abdominal pain, jaundice, and gastrointestinal hemorrhage, the complete typical triad can be seen in approximately two-thirds of patients who are usually presented with abdominal pain followed by hematemesis or melena [7]. In contrast, our patient was presented with jaundice and abdominal pain. It is possible that the gastrointestinal and biliary motility had been impaired due to the long-standing history of Parkinson's disease [26] so that the blood clots stayed longer in the common bile duct to represent the sign of jaundice first, and also in stomach and intestines to represent the late presentation of hematemesis and melena. On the other hand, abdominal US, especially color doppler US, has been recognized as a highly effective diagnostic tool for detecting pseudoaneurysms $[8,9]$. However, previous reports have demonstrated that a pseudoaneurysm was detected with color-doppler US only in 6 of 22 cases. Similarly, these imaging modalities were not sufficient enough to make a diagnosis in the present case because of the posture limitation and excessive bowel gas probably due to Parkinson's disease related impaired gastrointestinal motility.

In the present case, the patient was diagnosed with hemobilia confidently because the bleeding from the papilla of Vater was observed under direct endoscopic visualization by chance. If there was no chance to witness this finding, this patient would be diagnosed with acute gastric mucosal lesion, healed gastroduodenal ulcer, or any other cause of gastrointestinal bleeding, and undergo further re-intervention. It is noted that hemobilia should be included in the differential diagnosis of upper gastrointestinal bleeding when no obvious bleeding site can be found by upper endoscopy, and further imaging modalities such as contrast and/or dynamic CT and angiography should be considered to assess possible vascular abnormalities. DSA can be a both diagnostic and therapeutic method for the management of vascular abnormalities. Endoscopy can be a diagnostic tool but it is almost impossible to treat hemobilia with endoscopic procedure.
Hemobilia due to rupture of pseudoaneurysm developed on the cystic artery can be treated by either cholecystectomy or TAE. Maeda et al. [15] reported a case which was successfully treated by a "two-step" strategy consisting of embolization of the cystic artery followed by elective cholecystectomy. Whether cholecystectomy is required after successful embolization of cystic artery pseudoaneurysms remains controversial. Although the concern after embolization of cystic artery is the development of gallbladder ischemia, we assume that embolization of the cystic artery would not lead to ischemia of the gallbladder because it has been reported that the blood supply from gallbladder bed should be sufficient and collateral anastomoses with the epicholedochal artery may develop after embolization of the cystic artery [27]. In our case, we decided not to perform cholecystectomy following TAE because of severe co-morbidities and patient's refusal. Although cholecystectomy may not be required as far as there are no signs of gallbladder ischemia, the patient needs to be carefully monitored following TAE.

In conclusion, we report the case of hemobilia due to pseudoaneurysm originated from the cystic artery presenting with jaundice and upper abdominal pain followed by massive upper gastrointestinal bleeding, which was successfully treated by TAE. Hemobilia, especially due to psuedoaneurysm of cystic artery, is extremely rare. The direct visualization of blood from the papilla of Vater is crucial for the definitive diagnosis, but is difficult to encounter. Therefore, hemobilia should be included in the differential diagnosis of upper gastrointestinal bleeding with unknown etiology and there should be low threshold to perform dynamic CT and angiography to assess the vascular abnormalities. Although the management of hemobilia due to pseudoanerysm remains controversial, non-surgical approach such as TAE could be a reasonable and definitive treatment option especially for high-risk patients who are not suitable for surgery.

\section{Conflict of Interest}

Yoshihiro Komatsu and other co-authors have no conflict of interest.

\section{References}

1. Sandblom P. Hemorrhage into the biliary tract following trauma; traumatic hemobilia. Surgery. 1948;24(3):571586.

2. Green MH, Duell RM, Johnson CD, Jamieson NV. Haemobilia. Br J Surg. 2001;88(6):773-786.

3. Gutierrez G, Ramia JM, Villar J, Garrote D, Ferron A, Ruiz E. Cystic artery pseudoaneurism from an evolved acute calculous cholecystitis. Am J Surg. 2004;187(4):519-520. 
4. Morioka D, Ueda M, Baba N, Kubota K, Otsuka Y, Akiyama H, Endo I, et al. Hemobilia caused by pseudoaneurysm of the cystic artery. J Gastroenterol Hepatol. 2004;19(6):724-726.

5. Joyce MR, Donnolly M, O'Shea L, Jeffers M, O'Riordain D. Pseudoaneurysm of the cystic artery: a diagnostic dilemma and rare cause of haemobilia. Ir J Med Sci. 2006;175(1):81.

6. Lee JW, Kim MY, Kim YJ, Suh CH. CT of acute lower GI bleeding in chronic cholecystitis: concomitant pseudoaneurysm of cystic artery and cholecystocolonic fistula. Clin Radiol. 2006;61(7):634-636.

7. Sibulesky L, Ridlen M, Pricolo VE. Hemobilia due to cystic artery pseudoaneurysm. Am J Surg. 2006;191(6):797-798.

8. Akatsu T, Tanabe M, Shimizu T, Handa K, Kawachi S, Aiura K, Ueda M, et al. Pseudoaneurysm of the cystic artery secondary to cholecystitis as a cause of hemobilia: report of a case. Surg Today. 2007;37(5):412-417.

9. Saluja SS, Ray S, Gulati MS, Pal S, Sahni P, Chattopadhyay TK. Acute cholecystitis with massive upper gastrointestinal bleed: a case report and review of the literature. BMC Gastroenterol. 2007;7:12.

10. Machida H, Ueno E, Shiozawa S, Fujimura M, Tsuchiya A, Kim DH, Ogawa K, et al. Unruptured pseudoaneurysm of the cystic artery with acute calculous cholecystitis incidentally detected by computed tomography. Radiat Med. 2008;26(6):384-387.

11. Shimada K, Sakamoto Y, Esaki M, Kosuge T. Pseudoaneurysm of the cystic artery associated with xanthogranulomatous cholecystitis. Dig Surg. 2008;25(1):8-9.

12. Mullen R, Suttie SA, Bhat R, Evgenikos N, Yalamarthi S, McBride KD. Microcoil embolisation of mycotic cystic artery pseudoaneurysm: a viable option in high-risk patients. Cardiovasc Intervent Radiol. 2009;32(6):12751279.

13. Ahmed I, Tanveer UH, Sajjad Z, Munazza B, Azeem UD, Basit S. Cystic artery pseudo-aneurysm: a complication of xanthogranulomatous cholecystitis. $\mathrm{Br} \mathrm{J}$ Radiol. 2010;83(992):e165-167.

14. Hague J, Brennand D, Raja J, Amin Z. Cystic artery pseudoaneurysms in hemorrhagic acute cholecystitis. Cardiovasc Intervent Radiol. 2010;33(6):1287-1290.

15. Maeda A, Kunou T, Saeki S, Aono K, Murata T, Niinomi $\mathrm{N}$, Yokoi S. Pseudoaneurysm of the cystic artery with hemobilia treated by arterial embolization and elective cholecystectomy. J Hepatobiliary Pancreat Surg. 2002;9(6):755-758.

16. Rhee JW, Bonnheim DC, Upson J. Cystic artery pseudoaneurysm. N Y State J Med. 1987;87(1):47.

17. Wu TC, Liu TJ, Ho YJ. Pseudoaneurysm of the cystic artery with upper gastrointestinal hemorrhage. Case report. Acta Chir Scand. 1988;154(2):151-152.

18. Strickland SK, Khoury MB, Kiproff PM, Raves JJ. Cystic artery pseudoaneurysm: a rare cause of hemobilia. Cardiovasc Intervent Radiol. 1991;14(3):183-184.

19. Barba CA, Bret PM, Hinchey EJ. Pseudoaneurysm of the cystic artery: a rare cause of hemobilia. Can J Surg. 1994;37(1):64-66.

20. Nakajima M, Hoshino $H$, Hayashi E, Nagano $K$, Nishimura D, Katada N, Sano H, et al. Pseudoaneurysm of the cystic artery associated with upper gastrointestinal bleeding. J Gastroenterol. 1996;31(5):750-754.

21. England RE, Marsh PJ, Ashleigh R, Martin DF. Case report: pseudoaneurysm of the cystic artery: a rare cause of haemobilia. Clin Radiol. 1998;53(1):72-75.

22. Kaman L, Kumar S, Behera A, Katariya RN. Pseudoaneurysm of the cystic artery: a rare cause of hemobilia. Am J Gastroenterol. 1998;93(9):1535-1537.

23. Reddy SC. Pseudoaneurysm of cystic artery with upper gastrointestinal hemorrhage. South Med J. 1983;76(1):85-86.

24. Hakami M, Beheshti G, Amirkhan A. Hemobilia caused by rupture of cystic artery aneurysm. Am J Proctol. 1976;27(4):56-57.

25. Metcalfe D, Holt PJ, Thompson MM. The management of abdominal aortic aneurysms. BMJ. 2011;342:d1384.

26. Pfeiffer RF. Gastrointestinal dysfunction in Parkinson's disease. Lancet Neurol. 2003;2(2):107-116.

27. Takayasu K, Moriyama N, Muramatsu Y, Shima Y, Ushio K, Yamada T, Kishi K, et al. Gallbladder infarction after hepatic artery embolization. AJR Am J Roentgenol. 1985;144(1):135-138. 